\title{
Photodetector R\&D for the Belle II Upgraded Forward Electromagnetic Calorimeter
}

\section{G. Finocchiaro*}

Laboratori Nazionali di Frascati dell'INFN

E-mail: fnc@lnf.infn.it
A. Aloisio ${ }^{a, b}$,
S. Baccaro ${ }^{c, d}$, P. Branchini ${ }^{e}$,
S. Cavaliere ${ }^{a, b}$,
C. Cecchi ${ }^{f, g}$, A. Cemmi ${ }^{c, d}$,
G. Corradi ${ }^{h}$, E.
E. De Lucia ${ }^{h}$, G. De Nardo ${ }^{a, b}$, R. de Sangro ${ }^{h}$,
G. Felici ${ }^{h}$, S. Fiore ${ }^{c, d}$,
R. Giordano ${ }^{a, b}$, P. Lubrano ${ }^{g}$, E. Manoni ${ }^{g}$, A. Ordine ${ }^{b}$, A. Passeri ${ }^{e}$, P. Patteri ${ }^{h}$,
I. Peruzzi ${ }^{h}$, M. Piccolo ${ }^{h}, \mathbf{A}$. Rossi $^{g}$, C. Sciacca ${ }^{a, b}$, D. Tagnani ${ }^{e}$.
${ }^{a}$ Università degli Studi di Napoli "Federico II", Napoli, Italy;
${ }^{b}$ INFN Sezione di Napoli, Italy;
${ }^{c}$ ENEA UTTMAT, Roma, Italy;
${ }^{d}$ INFN Sezione di Roma, Roma, Italy;
e INFN sezione di Roma Tre, Roma, Italy;
${ }^{f}$ Università degli Studi di Perugia, Perugia, Italy;
${ }^{g}$ INFN Sezione di Perugia,Italy;
${ }^{h}$ Laboratori Nazionali di Frascati dell'INFN, Frascati, Italy.

The Belle II experiment will operate at the SuperKEKB $e^{+} e^{-}$collider, designed to reach a peak luminosity of $8 \times 10^{35} \mathrm{~cm}^{-2} \mathrm{~s}^{-1}$ at the $\Upsilon(4 S)$ resonance. The high background environment of SuperKEKB poses serious challenges to the design of the Belle II detector. In particular, an upgrade of the forward Electromagnetic Calorimeter is foreseen: the new calorimeter will use pure CsI crystals, which have a faster scintillation light emission and lower light yield than the CsI(Tl) crystals presently used. An intense R\&D program is ongoing to select the optimal photodetector to meet the stringent requirements set by the use of pure CsI in Belle II. A study of equivalent noise, resolution, radiation hardness and stability of low noise, high-gain avalanche photodiodes obtained by reading single pure CsI crystals is discussed. Our preliminary results indicate that a readout chain using these devices meets the requirements on electronic noise set by the Belle II simulation and could represent a cost-effective choice for the readout of pure CsI crystals in general.

Technology and Instrumentation in Particle Physics 2014,

2-6 June, 2014

Amsterdam, the Netherlands

*Speaker. 


\section{Introduction}

Motivated by the enormous impact the $B$ Factories had in High Energy Physics in the past decade, the $e^{+} e^{-}$collider at KEK, Japan has been redesigned to reach the peak luminosity of $8 \times$ $10^{35} \mathrm{~cm}^{-2} \mathrm{~s}^{-1}$ at the $\Upsilon(4 S)$ resonance (SuperKEKB). The Belle detector [1] is also being upgraded in order to maintain its performance in the presence of considerably higher background levels.

The Belle II Technical Design Report [2] describes in detail the modifications to the various subdetectors; in particular, for the Tallium-doped CsI crystals of the electromagnetic calorimeter, the concern exists of radiation damage and of high pileup levels, especially at small polar angles. For this reason, at least part of the crystals of the forward electromagnetic calorimeter will be replaced with pure CsI crystals. Undoped CsI has in fact improved radiation hardness, and much faster light decay time (6/35 ns for the two main components, compared to $1.2 \mu \mathrm{s}$ in $\mathrm{Cs}(\mathrm{Tl})$ ) [3].

However, pure CsI features low light yield, and has the emission peak of the fast components at $310-420 \mathrm{~nm}$ wavelengths. The PIN photodiodes reading the present $\mathrm{Cs}(\mathrm{Tl})$ crystals must therefore be replaced by photodetectors best matching these characteristics, that is having large internal gain, and good efficient in the near-UV region.

The present baseline envisages a 2-inch photopentode by Hamamatsu Photonics [4] for its large signal. This device is however quite sensitive to the magnetic field, would offer no redundancy in the event of failure and, due to its relatively large size, would require rebuilding the supporting structure for the electronics. An alternative readout option, which would address these two issues, would be the use of much more compact devices such as Avalanche Photo Diodes (APDs). This solution is under study; in this paper we discuss preliminary results from R\&D work on two different Avalanche Photodiode types: the C30739ECERH-2 from Excelitas Technologies [5] (hereafter referenced as ET-APD), and the S8664-1010 from Hamamatsu Photonics [4] (HP-APD in the following).

The former features high gain, $G=200$, at the nominal bias voltage $V_{\text {nom }}$, small capacitance $(\simeq 60 \mathrm{pF})$, very small dark current $(\leq 1 \mathrm{nA})$ and good spectral response at the relevant wavelengths of $\lambda \geq 310 \mathrm{~nm}$. As the photodetector active area is relatively small $\left(5.6 \times 5.6 \mathrm{~mm}^{2}\right)$, the crystals must be instrumented with more than one APD; we used four in our research.

The HP-APDs are larger $\left(10 \times 10 \mathrm{~mm}^{2}\right)$ - which allowed us to equip the crystals with only two of them. However, they have higher capacitance $(\simeq 270 \mathrm{pF})$ and lower nominal gain of $G=50$ than the ET-APDs. We found that the needed signal to noise ratio $(\mathrm{S} / \mathrm{N})$ could be achieved for a gain of $G=200$, which we obtained by increasing the operating bias voltage. The dark current at this gain has been measured to be between 15 and $20 \mathrm{nA}$.

These two options have been explored and the results will be reported in the rest of this paper.

\section{Experimental setup}

The response of the two types of photosensors to the light emitted by pure CsI crystals has been studied independently in two different laboratories, with similar experimental setups. Both setups include a light-tight box with temperature and humidity monitors. The relative humidity in the boxes is maintained below $\mathrm{RH} \simeq 15 \%$ by flushing dry air; the room temperature is kept constant within $\simeq 2^{\circ} \mathrm{C}$ by conventional air conditioning systems. In each setup a cosmic-ray telescope 
was assembled with a pair of scintillator counters placed above and below the crystal under study. The telescope geometry is such that the selected tracks are almost vertical. Since in the present discussion we are only interested in the mean value of the signal, the residual $\mathscr{O}(10 \%)$ RMS spread of the track length inside the crystal does not introduce any bias.

The CsI crystals used in the tests, with size $5 \times 5 \times 30 \mathrm{~cm}^{3}$ similar to those used in the Belle calorimeter, were produced by Amcrys [6]; they are wrapped in Tyvek paper and then with black tape. The APDs are optically coupled to the rear face of the crystal using BC630 Saint-Gobain silicon optical grease. The photosensors are then readout using a CREMAT [7] CR-110 low-noise commercial amplifier, with a gain of $1.4 \mathrm{mV} / \mathrm{fC}$, mounted on a CR-150 board by CREMAT. For the ET-APDs a custom board housing four CR-110 amplifiers was designed. The amplified signals are recorded by a digital oscilloscope [8], with 1GS/s sampling frequency.

\section{Results}

Based on simulation studies of the expected background rates in the Belle II forward endcap calorimeter [2], in order to have a negligible electronics noise contribution with respect to the pileup noise, the Equivalent Noise Energy (ENE) should be kept below $1 \mathrm{MeV}$.

As reference, we measured the performances of the 2-inch photopentode (PP), the Hamamatsu R11283, presently chosen as baseline for the the Belle II calorimeter upgrade. A charge amplifier with gain of $1.4 \mathrm{mV} / \mathrm{fC}$ and $\tau=150 \mathrm{~ns}$ was developped to readout the PP. We exposed a single CsI crystal to a $100 \mathrm{MeV}$ electron beam at the Frascati Beam Test facility [9]. Simulation studies show that about $80 \%$ of the shower energy is released inside the crystal; with this normalization, the electronic noise measured by the PP corresponds to $\mathrm{ENE} \simeq 220 \mathrm{keV}$, which fully meets the requirement.

In the following sections, we discuss separately the studies performed on the ET-APDs and HP-APDs, and finally a simultaneous test of the two.

\subsection{Excelitas C30739ECERH-2}

Gain measurements as a function of the APD operating voltage have been performed illuminating the device with an LED. The observed gain is consistent with the manufacturer's specifications

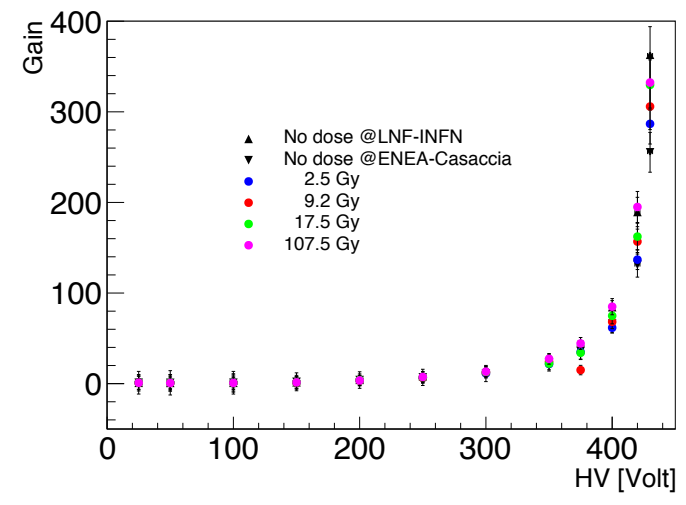

Figure 1: APD gain curves for different values of integrated $\gamma$ rates. 
$\left(G \simeq 200\right.$ for $\left.V_{\text {bias }}=420 \mathrm{~V}\right)$. A radiation hardness test was also carried out, by irradiating the APD with $1.25 \mathrm{MeV}$ photons at the ENEA-Casaccia "Calliope" facility [10]. Fig. 1 shows the measured gain as a function of the integrated dose. No appreciable variations are observed within the sensitivity of our setup up to $100 \mathrm{~Gy}$, the $\gamma$ dose expected in the hot regions of the Belle II forward electromagnetic calorimeter during the experiment lifetime.

A typical recorded waveform from a cosmic ray track traversing the crystal in the setup is shown in Fig. 2 (left). To remove fluctuations of the baseline, the signal amplitude is obtained
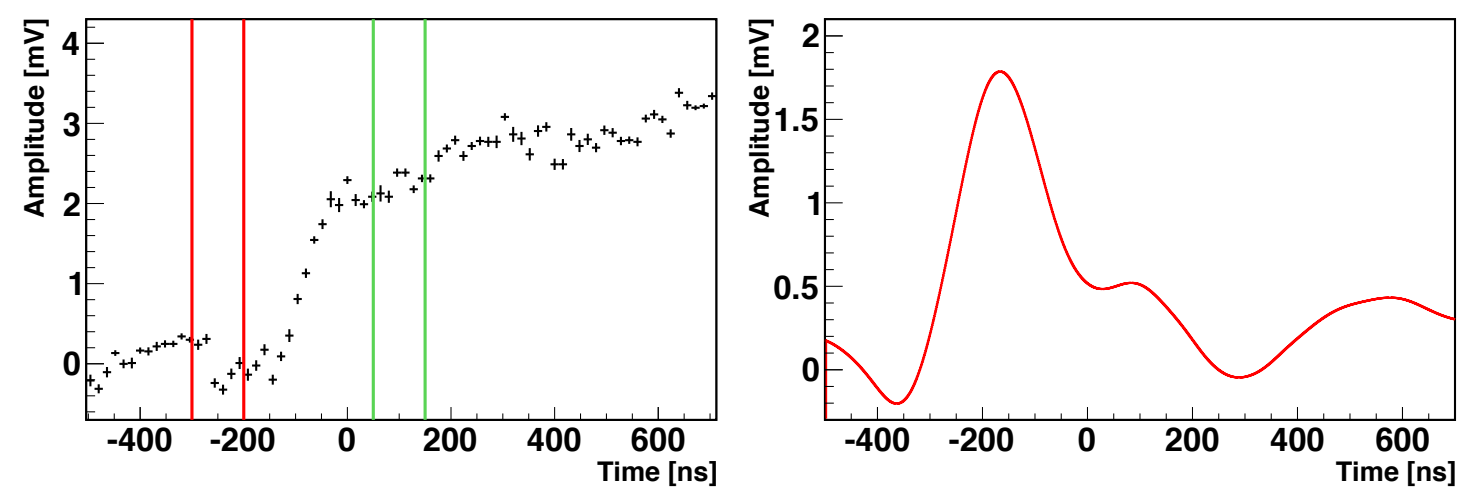

Figure 2: Signal from one of the four ET-APDs reading a CsI crystal. Left: raw signal - the vertical lines indicate the intervals used to sample the signal. Right: output of a CR-(RC) ${ }^{4}$ software filter with $\tau=75 \mathrm{~ns}$.

by taking the difference of the waveform sampled immediately after and before the trigger (double sampling). Fig. 2 (right) shows the same waveform after application of a CR-(RC) ${ }^{4}$ filter, with $75 \mathrm{~ns}$ time constant. In this case we consider as signal amplitude the maximum of the shaped waveform.
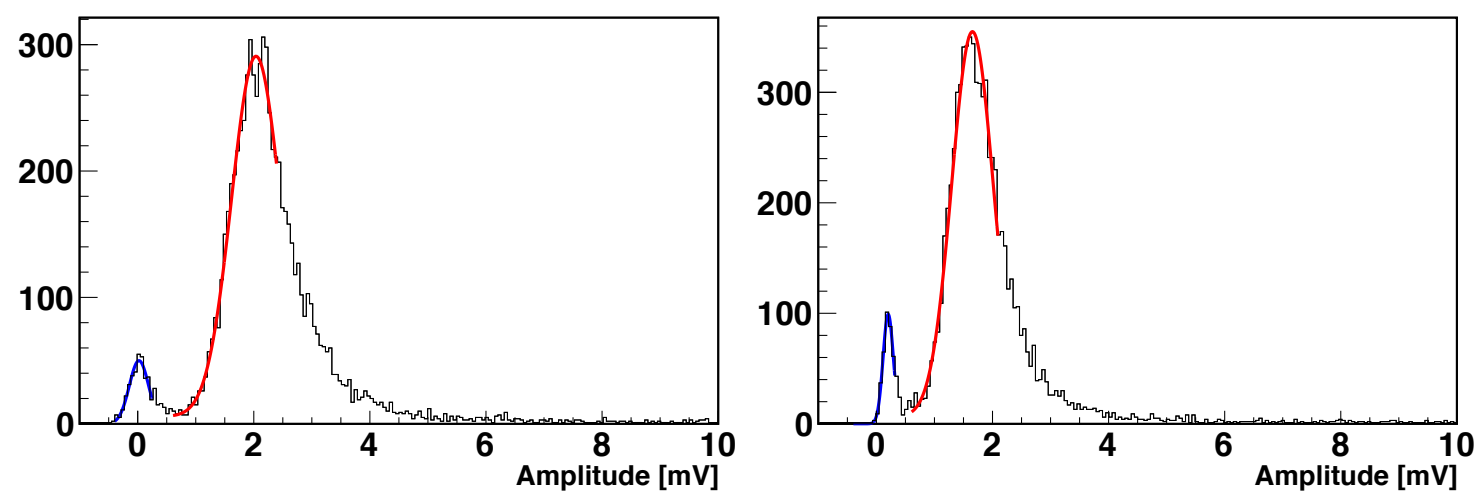

Figure 3: Signal amplitude of one of the four ET-APDs. Left: double sampling of the raw waveform. Right: $\mathrm{CR}-(\mathrm{RC})^{4}$-shaped waveform with $\tau=75 \mathrm{~ns}$.

The resulting amplitudes for one of the four APDs instrumenting the crystal are shown in Fig. 3, double sampling the raw waveform (left) and after applying the filtering algorithm (right).

The equivalent noise energy is proportional to the RMS of the pedestal. The energy scale is set by the position of the signal peak, which corresponds to the average energy released by a cosmic ray track in the $5 \mathrm{~cm}$ thick crystal $(30 \mathrm{MeV})$. We perform fits to the distributions in Fig. 3 to measure position and width of the pedestal peak (the blue curve superimposed to the histogram), 
and the position of the signal peak (red curve). The improvement obtained shaping the waveform is evident: the pedestal width in the right-hand plot is considerably more reduced than the signal amplitude. Indeed, for a single APD we obtain $\mathrm{ENE}=(2.78 \pm 0.19) \mathrm{MeV}$ with double sampling, and $\mathrm{ENE}=(1.79 \pm 0.12) \mathrm{MeV}$ after shaping.

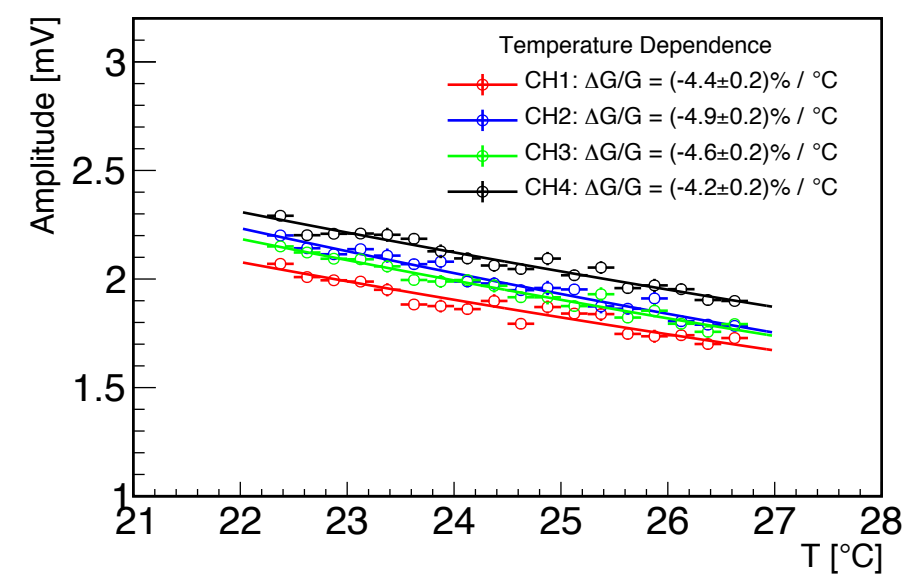

Figure 4: Dependence of signal amplitude on temperature. The measured value of $\Delta G / G$ includes the contribution $\simeq-1.5 \%$ of the pure CsI crystal.

In order to quantify the temperature dependence of the ET-APD APD response, we have extended the range of temperatures during data taking by temporarily switching off the air conditioning in the laboratory. The observed gain variation is illustrated in Fig. 4 for a set of four APDs. We measure an average coefficient $\Delta G / G=-4.5 \pm 0.2 \% /{ }^{\circ} \mathrm{C}$. Part of this variation is due to the light yield variation of the CsI crystal of about $-1.5 \% /{ }^{\circ} \mathrm{C}[11,12]$.

In the following we normalize the measured amplitudes to $25^{\circ} \mathrm{C}$, which was the average operation temperature of the forward endcap calorimeter during the data taking in Belle.

If the APD intrinsic noise is uncorrelated, it is convenient to sum the individual amplitudes. As an example, results obtained operating the four APDs at their nominal bias voltage are shown in Table 1, which details the measured noise, signal levels, S/N and ENE values for each of the four APDs and for their sum, when a software shaper with time constant $75 \mathrm{~ns}$ is applied. As expected, the ENE of the sum improves by a factor 2 compared to the single APDs.

\begin{tabular}{|ccccc|}
\hline \hline APD & $\sigma[\mu \mathrm{V}]$ & Signal $[\mathrm{mV}]$ & S/N & ENE $[\mathrm{MeV}]$ \\
\hline 1 & $55 \pm 3$ & $1.45 \pm 0.01$ & $26 \pm 1$ & $1.15 \pm 0.06$ \\
2 & $59 \pm 3$ & $1.39 \pm 0.01$ & $24 \pm 1$ & $1.28 \pm 0.06$ \\
3 & $85 \pm 4$ & $1.33 \pm 0.01$ & $16 \pm 1$ & $1.92 \pm 0.09$ \\
4 & $73 \pm 3$ & $1.37 \pm 0.01$ & $19 \pm 1$ & $1.60 \pm 0.07$ \\
\hline SUM & $174 \pm 8$ & $5.35 \pm 0.02$ & $31 \pm 1$ & $0.98 \pm 0.05$ \\
\hline \hline
\end{tabular}

Table 1: Measured quantities for the four ET-APDs, and their sum.

To avoid the complication of reading four APDs for each crystal, we have studied how effective is to sum the signals before amplification. We made a simple analog sum of the signals from two APDs, and recorded them. We have then compared the software sum of these two signals with 
that of the four individual APD signals. Using the standard double sampling analysis we obtained: $\mathrm{ENE}=(1.69 \pm 0.16) \mathrm{MeV}$ for the analog sum, and $\mathrm{ENE}_{\text {digital }}=(1.63 \pm 0.09) \mathrm{MeV}$ for the sum of four APDs, which are in perfect agreement.

\subsection{Hamamatsu S8664-1010}

Gain measurements performed for the HP-APD by illuminating them with an LED are shown in Fig. 5. These curves are used for adjusting the bias voltage to operate the devices at gain $G=200$.
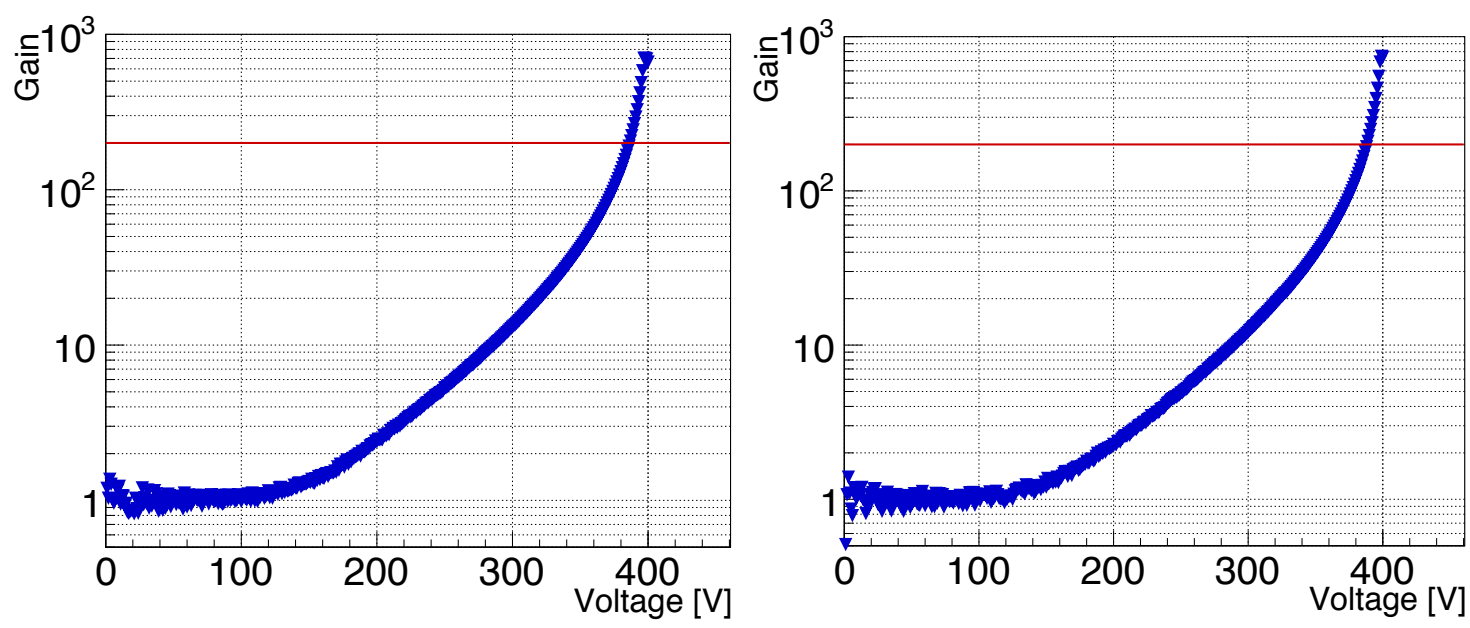

Figure 5: Gain curve measured illuminating with an LED two of the Hamamatsu S8664-1010 APDs.

The response of the two HP-APDs to cosmic-ray particles, with no shaping applied, is shown in Fig. 6. The ENE values measured for the two APDs are $\mathrm{ENE}_{1}=(1.9 \pm 0.1) \mathrm{MeV}$ and $\mathrm{ENE}_{2}=$ $(2.3 \pm 0.1) \mathrm{MeV}$ respectively. Summing the amplitudes we get $\mathrm{ENE}_{\mathrm{SUM}}=(1.6 \pm 0.1) \mathrm{MeV}$. As
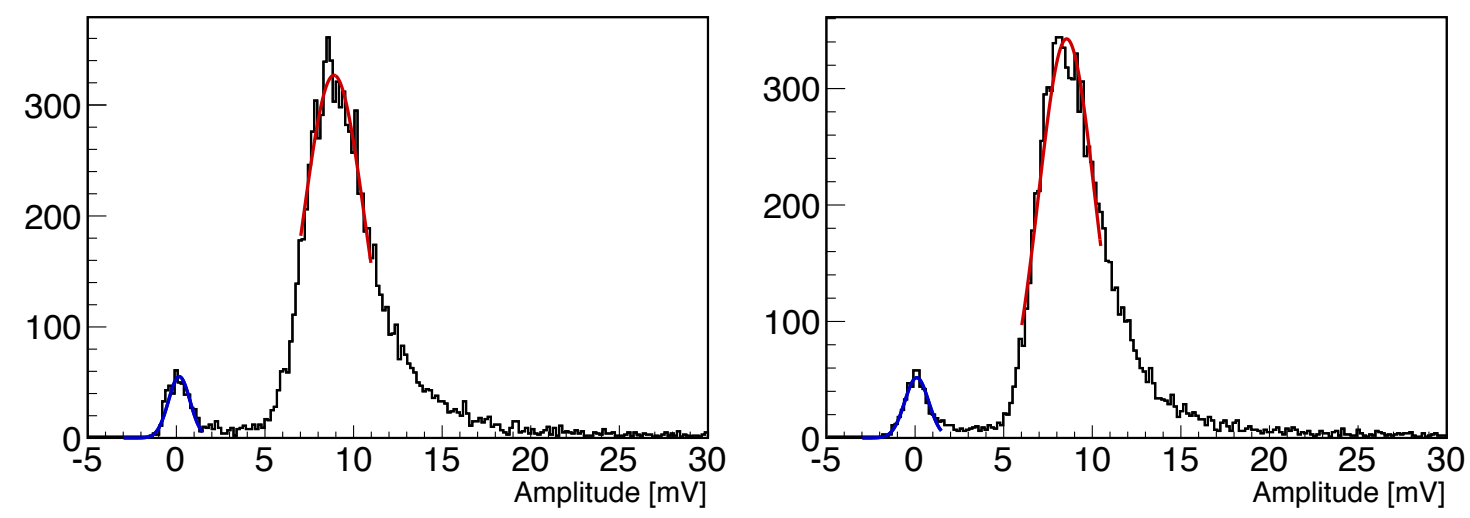

Figure 6: Raw signal amplitudes of the two HP-APDs.

for the ET-APDs, the $\mathrm{S} / \mathrm{N}$ is much improved when a filtering algorithm is applied, a trapezoidal shaping with $75 \mathrm{~ns}$ time constant in this case. The result is $\mathrm{ENE}_{\mathrm{SUM}}=(0.9 \pm 0.1) \mathrm{MeV}$.

The temperature dependence of the gain of one HP-APD sample, shown in Fig. 7 (left), was obtained in a dedicated measurement using a thermalized chamber. The temperature coefficient 
was measured very precisely to be $\Delta G / G=(-4.16 \pm 0.04) \% /{ }^{\circ} \mathrm{C}$, consistent with the one for the ET-APDs.

In the setup used for the HP-APDs, the readout chain can optionally be equipped with a shaping amplifier (CREMAT CR-200) with time constant 100 ns. In Fig. 7 (right) we show the temperature dependence of the equivalent noise energy obtained with this hardware shaper. As one can see, the ENE measured at $25^{\circ} \mathrm{C}$ of about $0.9 \mathrm{MeV}$ is comparable with the value obtained with the software filter.
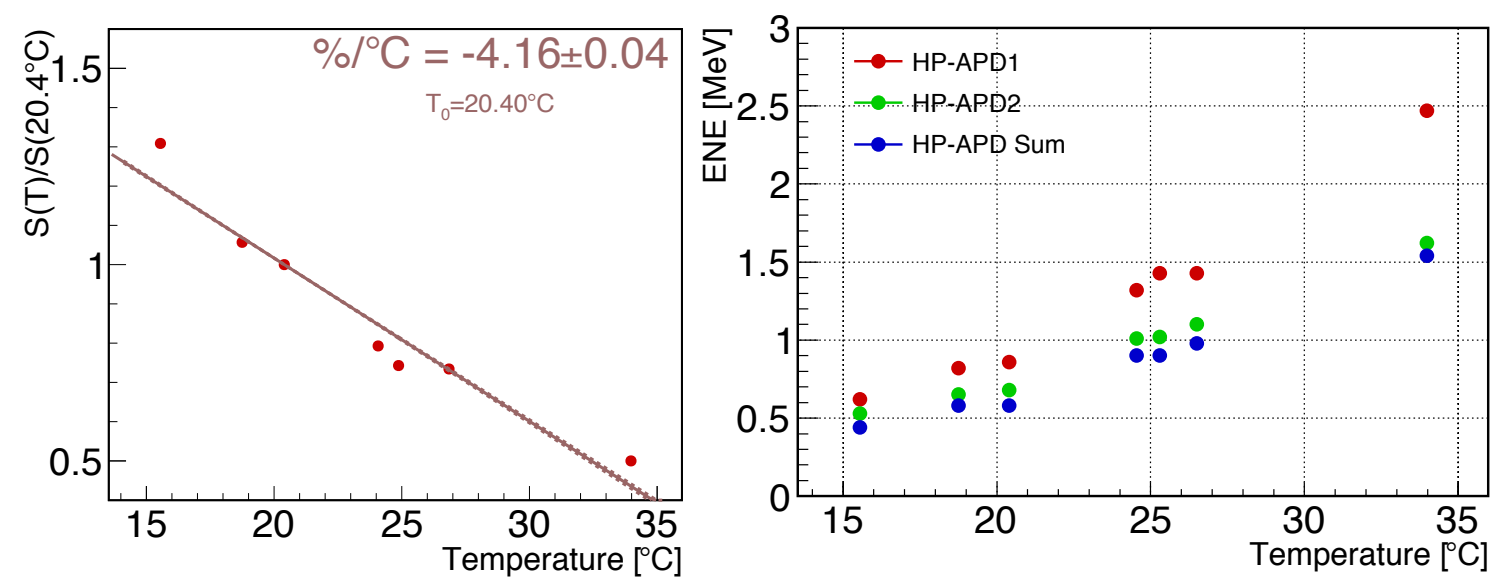

Figure 7: Left: signal amplitude vs. temperature for one HP-APD. Right: equivalent noise energy vs. temperature for two APDs and their sum obtained with the CREMAT shaping amplifier.

\subsection{Direct APD comparison}

In order to make a comparison of the two types of APDs in the very same experimental conditions (light yield of the detection crystal, environmental noise, temperature), we equipped one crystal with the two HP-APDs on one face, and four ET-APDs (a different set from the one we discussed in Table 1) on the opposite face. We define $V_{\text {nom }}$ as the voltage at which $G=200$, and $\Delta V=V_{\text {bias }}-V_{\text {nom }}$ the difference with the applied bias voltage $V_{\text {bias. }}$ In Fig. 8 (left) we show the sig-
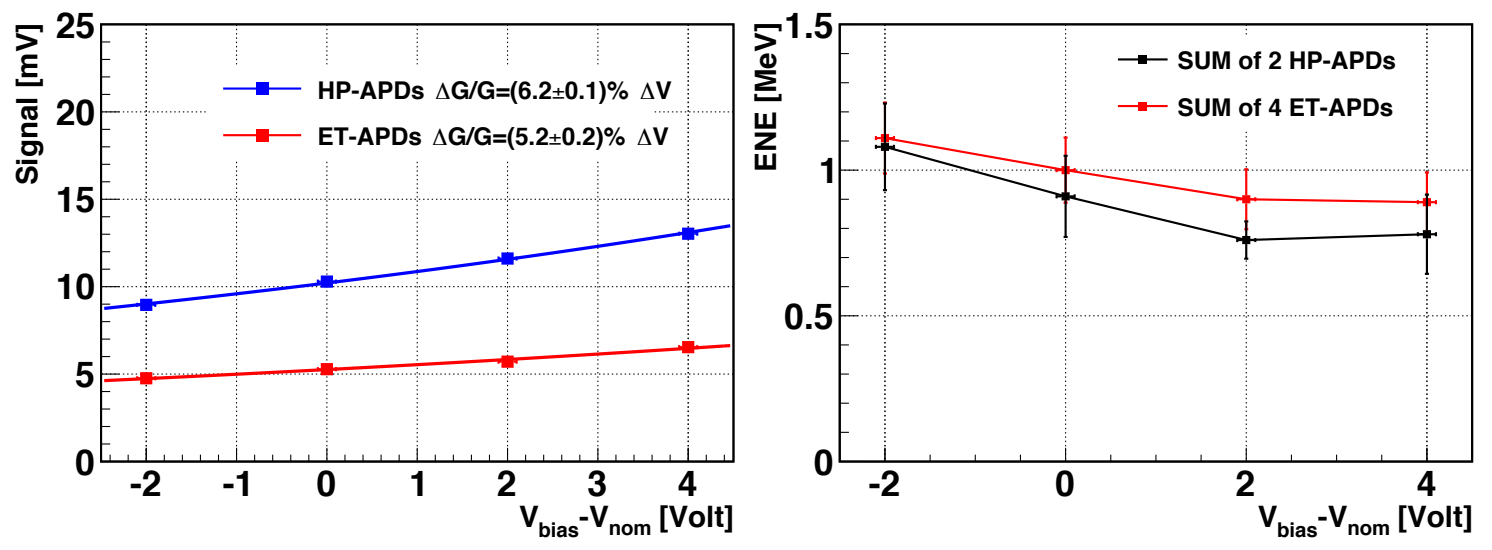

Figure 8: Sum of the 4 ET-APDs or of the 2 HP-APDs mounted on the same crystal as a function of

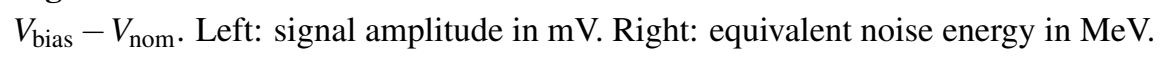


nal amplitude as a function of $\Delta V$ for the sum of four ET-APDs and for the sum of two HP-APDs. The $\Delta G / G$ coefficients are quite similar for the two devices. On the right-hand side of the same figure we show the equivalent noise energy again as a function of $\Delta V$. The expected trend of ENE decreasing with increasing bias voltage is qualitatively observed. The ET-APDs and the HP-APDs show similar performances within our experimental uncertainty, and both meet the requirement $\mathrm{ENE} \leq 1 \mathrm{MeV}$.

\section{Summary}

Preliminary results are discussed of an R\&D being conducted in the contest of the upgrade of the Belle II forward endcap calorimeter. The upgrade foresees to replace part of the detector Cs(Tl) crystals with undoped CsI crystals to control radiation damage and the deterioration of energy resolution due to pile-up. Photodetectors to readout such crystals must match the properties of CsI, in particular have large internal gain, good spectral response down to $\lambda \simeq 310 \mathrm{~nm}$, and small intrinsic noise.

Two different APD types have been used to read out the scintilation light of pure CsI: the C30739ECERH-2 from Excelitas (four sensors per crystal) and the S8664-1010 from Hamamatsu (two sensors per crystal). Both APD types have been operated at gain $\mathrm{G}=200$. The gain variation with the operation temperature was measured to be $\Delta G / G \simeq-3 \% /{ }^{\circ} \mathrm{C}$ for both photodiodes. The gain variation with the bias voltage is also similar for the two devices, $\Delta G / G \simeq 5 \% /$ Volt.

With the readout electronics used for the present study, the summed amplitudes of both APD types meet the requirements on electronic noise set by Belle II simulation studies, ENE $\leq 1 \mathrm{MeV}$, with similar performances: the higher responsivity in the $300-400 \mathrm{~nm}$ range and much smaller dark current of the UV-enhanced ET-APDs balance their active area being 60\% smaller than HP-APDs.

Irradiation tests made on the ET-APDs gave preliminary indication that these devices are insensitive to $\gamma$ doses up to at least $100 \mathrm{~Gy}$. Similar tests are being performed on the HP-APDs. Additional studies on possible damage under neutron irradiation are ongoing.

\section{Acknowledgments}

We gratefully acknowledge the dedication and technical skills of M. Bizzarri, A. Papi, A. Russo, G. Scolieri and A. Zossi.

\section{References}

[1] A. Abashian et al., Nuclear Instruments and Methods A479 (2002), 117.

[2] T. Abe et al., Belle II Technical Design Report. arXiv:1011.0352 [physics.ins-det].

[3] R-y. Zhou, G. Gratta, H. Newman, Nucl. Phys. B (Proc. Suppl.) 44 (1995), 88-108.

[4] http://www.hamamatsu.com

[5] http://www.excelitas.com/Pages/Product/Photonic-Detectors.aspx

[6] http://www.amcrys.com

[7] http://www.cremat.com 
[8] http://www.teledynelecroy.com

[9] G. Mazzitelli et al., DAФNE Beam Test Facility commissioning. Nucl. Instr. Meth. A515 (2003), 524.

[10] S. Baccaro, A.Cemmi, Radiation damage studies performed at the Calliope gamma irradiation plant at ENEA (Italy). SPIE Proceedings Vol. 8144.

[11] http://pdg.lbl.gov/2013/reviews/rpp2013-rev-particle-detectors-accel.pdf

[12] http://www.crystals.saint-gobain.com/uploadedFiles/SG-Crystals/Documents/CsI Pure Data Sheet.pdf 